\title{
MARÍA ISABEL TORRES CAZORLA: LA "NUEVA" AMENAZA DE CÓLERA EN HAITÍ TRAS EL PASO DEL HURACÁN MATTHEW: ¿HAY RESPUESTA DESDE EL CONTEXTO ONUSIANO?
}

\section{"THE "LATEST"' THREAT OF CHOLERA IN HAIITI AFTER HURRICANE MATTHEW:DOES THE UN HAVE A REACTION?"}

Resumen: En este trabajo se pretende abordar, de manera breve, lo acontecido respecto de la epidemia de cólera desatada en Haití durante el año en curso, de la que ya existían indicios en el momento en que se estaba desarrollando el plenario de la Asamblea General en el actual período de sesiones. Por supuesto, la situación se ha agravado de forma sustancial tras el paso del huracán Matthew y la devastación provocada por el mismo. Desde un contexto conectado con la salud como derecho humano, el bioderecho y las eventuales respuestas que Naciones Unidas y su familia pueden ofrecer, cabe preguntarnos por las eventuales respuestas que se están dando desde dichos organismos internacionales para hacer frente a este desafío.

Abstract: Last September 2016 during the Annual General Debate of the United Nations General Assembly the issue of health risks in the wake of natural disasters like hurricane Matthew arose. Undoubtedly, basic existing problems with health care have been dramatically compounded as a consequence of hurricane "Matthew". This paper focuses on the cholera epidemic in Haiti at the end of this year using the perspective of the right to health as an essential human right, biolaw and UN response. There is a key question that must be addressed- How can these international entities deal with these challenges?

Palabras clave: Catástrofes, Cólera, Derecho a la salud, Haití, Naciones Unidas, Organismos especializados.

Key words: Cholera, Disasters, Haiti, Right to health, Specialized Agencies, United Nations.

\section{Introducción}

El presente trabajo intenta poner en conexión varias materias que guardan una relación intrínseca, aunque a primera vista pudiera no parecerlo: por un lado, el ámbito de los derechos humanos (conectado directamente con el estudio de la situación dramática que vive -desde hace ya décadas- un 
Estado como Haití y su población). Se trata de la epidemia de cólera-que ya parece instaurada como un mal endémico en dicho territorio desde hace décadas- y su rebrote en los últimos tiempos, agravado éste por lo acontecido tras el huracán Matthew que ha azotado las costas norteamericanas en este mes de octubre de 2016. Cierto es, y a ello obedece el calificativo de "nuevo" entre comillas con el que hemos rotulado el título, que como veremos, ya existían indicios sobrados del rebrote de dicha enfermedad en el mes de septiembre -e incluso con anterioridad- como se puso de relieve el día 23 de dicho mes por parte del Presidente en funciones haitiano -Jocelerme Privert- al dirigirse en su discurso al pleno de la Asamblea General de Naciones Unidas. Pero, que duda cabe que el paso del huracán Matthew ha unido a la oleada de devastación (en términos humanos y materiales) un incremento mayor de propagación de dicha enfermedad. Junto con los derechos humanos, la respuesta por parte de los organismos internacionales (especialmente de Naciones Unidas y sus organismos especializados y agencias) ante una situación catastrófica como la antedicha, será el eje fundamental sobre el que gire este breve estudio. Y ello con el objetivo de responder a una cuestión, tal vez irresoluble: ¿están capacitados estos entes internacionales para afrontar situaciones dramáticas como las que vive Haití -y no hay que olvidar que no es la primera vez que ocurre una situación de dimensiones similares en este mismo Estado- con el objetivo de que, a los muertos provocados por las catástrofes naturales no haya que sumar otros cientos o miles producidos por la expansión de una epidemia de cólera como la que ya está surgiendo?

\section{Nada "nuevo" bajo el sol: la historia se repite}

Posiblemente, cualquier persona que realice un acercamiento, siquiera sea consultando los medios de comunicación y la atención prestada a la "realidad haitiana" de los últimos años, llegará a una conclusión sencilla: la situación vivida por esta isla (compartida con otro Estado, la República Dominicana) resulta dramática. Esa situación que cabría calificar como "desastrosa y caótica" obedece tanto a la inestabilidad política, social, como a la propia realidad que allí se vive, al ser un territorio azotado por cataclismos de muy diversa índole (terremotos y zona de paso de huracanes en cada temporada en que ese fenómeno tiene lugar). La pobreza, junto con la inestabilidad política ${ }^{1}$ conforman un peligroso cocktail que, aunado, lleva a una situación gravísima como la que vive Haití, podemos decir que prácticamente desde el comienzo de los tiempos ${ }^{2}$.

\footnotetext{
${ }^{1}$ Vid. M.I. TORRES CAZORLA, "Environmental Security: an Initial View from the Perspective of International Law", en La seguridad internacional en el Siglo XXI. Nuevas Perspectivas, E.M GARCÍA RICO y M.I. TORRES CAZORLA (coords.), Plaza y Valdés, Madrid, 2011, pp. 145166, en particular pp. 153-154, permitiéndonos calificar como "catastrophic countries" a Haití y otros Estados del mundo que viven situaciones de inestabilidad en tantos frentes.

${ }^{2}$ Un análisis de los principales hitos por los que ha discurrido la historia -y sobre todo las desventuras de este territorio desde su conquista en 1492-, la erradicación de su población nativa y su desplazamiento por esclavos traídos del continente africano puede verse en el
} 
Han sido diversas las ocasiones en que desde hace años Haití ha sido asolado por el cólera; inclusive, como factor que ha contribuido aún más a agravar la situación, la acusación a la MINUSTAH ${ }^{3}$ de haber sido un elemento "coadyuvante" para la expansión de la enfermedad desde la década pasada ${ }^{4}$, así como los procedimientos judiciales abiertos en Estados Unidos sobre el particular" ${ }^{5}$, son factores que traen a la mente la manida frase de "la historia se repite". El devastador terremoto sufrido en 2010, junto al brote de cólera desatado de forma desaforada en octubre de ese año constituyen antecedentes que hacen presagiar lo peor respecto a la tragedia que se vive en este último trimestre de 2016, tras el paso del huracán Matthew. Como dice el refrán, "llueve sobre mojado". Son diversos los indicios que nos dan pistas de esta realidad, de los que vamos a mostrar tres ejemplos. Así, en octubre de 2015, el Consejo de Seguridad, en su Resolución 2243, ponía de relieve la situación, que lejos de tranquilizar, señalaba la dificultad que suponía erradicar el cólera del territorio haitiano, pese a los esfuerzos realizados hasta ese momento:

análisis que realizan S. VON EINSIEDEL y D.M. MALONE, "Haiti", en The UN Security Council. From the Cold War to the 21st Century, D.M. MALONE (ed.), Lynne Rienner Publishers, Boulder, London, 2004, especialmente en pp. 467-468. El enorme número de constituciones y de Jefes de Estado desde el siglo XIX, en palabras de dichos autores -21 y 42, respectivamente a comienzos del siglo XXI-constituyen una buena muestra de la inestabilidad en el terreno político, que se extrapola a otros órdenes.

${ }^{3}$ Vid. toda la información relativa a la MINUSTAH, esto es, la Misión de Estabilización de las Naciones Unidas en Haití, establecida el 1 de junio de 2004 por la Resolución del Consejo de Seguridad 1542, de 30 de abril de dicho año, y que sustituyó a la previa Fuerza Multinacional Provisional que autorizó el Consejo de Seguridad unos meses antes, en febrero de 2004, en http://www.un.org/es/peacekeeping/missions/minustah/. Este enlace, así como todos los citados en este trabajo, han sido consultados por última vez el 7 de noviembre de 2016.

${ }^{4}$ La bibliografía relativa a la expansión del cólera y el papel jugado por las fuerzas de Naciones Unidas y su eventual responsabilidad es muy extensa; simplemente, a modo ejemplificativo, pueden consultarse, entre otros, MÉGRET, F., "La responsabilité des Nations Unies aux temps du chólera", 47 Revue Belge de Droit International (2013-1), pp. 161-189; TAYLOR, K.N., "Shifting Demands in International Institutional Law: Securing the United Nations' Accountability for the Haitian Cholera Outbreak", 45 Netherlands Yearbook of International Law (2014), pp. 157-195; HOLLENBERG, S., "Immunity of the UN in the case of Haitian Cholera Victims", 19 Journal of International Peacekeeping (2015, 1 y 2), pp. 118-141; PAVONI, R., "Choleric notes on the Haiti Cholera Case", Questions of International Law (27 July 2015), pp. 11-37, accesible en http://www.qil-qdi.org/choleric-notes-on-the-haiti-cholera-case/; BODE, T.G., "Cholera in Haiti: United Nations Immunity and Accountability", 47 Georgetown Journal of International Law (2016-2), pp. 759-791. Como M.P. SNYDER puso de relieve en 2014, "the cholera epidemic in Haiti, in which Nepali peacekeepers are believed to have introduced the disease into the country, has underscored the need for a better understanding of the linkages -both good and bad- between health and peacekeeping" (vid. https://theglobalobservatory.org/2014/12/securitycouncil-response-ebola-action/).

${ }^{5}$ Si bien en agosto de 2016 el Tribunal de Apelaciones reconoció la inmunidad de las Naciones Unidas, manteniendo la posición previamente sostenida por un juez federal de Nueva York, el Secretario General de Naciones Unidas, Ban Ki-moon, afirmó que la organización "tiene una responsabilidad moral con las víctimas de la epidemia de cólera y para ayudar a Haití a superar la epidemia y construir sistemas de agua, saneamiento y salud sólidos". Vid. la noticia de prensa relativa al fallo del Tribunal de apelaciones en http://www.nytimes.com/2016/08/19/world/americas/united-nations-cholera-haiti.html? $\quad r=0$. 
"Acogiendo con beneplácito los esfuerzos del Gobierno de Haití por controlar y erradicar la epidemia de cólera, los progresos realizados en la reducción de la incidencia del cólera en Haití, instando al equipo de las Naciones Unidas en el país a que, en coordinación con otras instancias, siga apoyando al Gobierno de Haití para solventar las deficiencias estructurales, particularmente en los sistemas de suministro de agua y saneamiento, recalcando la importancia de reforzar las instituciones nacionales haitianas de salud, reconociendo los esfuerzos de las Naciones Unidas por luchar contra el cólera, incluso mediante la iniciativa del Secretario General para apoyar el Plan Nacional para la Eliminación del Cólera, destacando la importancia de que se ofrezca un apoyo adecuado y sostenible prestando especial atención a las respuestas médicas rápidas y específicas contra los brotes de la enfermedad destinadas a reducir la amenaza, recordando la visita que realizó el Secretario General a Haití en julio de 2014, y tomando nota de que, entre otras cosas, anunció junto al ex Primer Ministro la "Campaña de Saneamiento Total", iniciativa clave contra el cólera, y la creación del Comité de Alto Nivel para la Eliminación del Cólera,

Acogiendo con beneplácito la primera reunión del Comité de Alto Nivel para la Eliminación del Cólera desde el cambio de Gobierno en enero de 2015, presidida conjuntamente por el Primer Ministro, Evans Paul, y la Representante Especial del Secretario General, Sandra Honoré, y observando que el aumento de los casos de cólera registrado a finales de 2014 y en los primeros cuatro meses de 2015 comenzó a mostrar signos de remisión en mayo, a pesar de que la situación sigue siendo preocupante en los departamentos del Oeste, Centro, Artibonite y Norte, en los que se concentró más del $80 \%$ del número total de casos entre marzo y agosto de $2015(\ldots)^{\prime \prime 6}$.

En noviembre de ese mismo año 2015, había al menos quince comunidades declaradas en "alerta roja" por las autoridades, ante el brote de cólera en el país ${ }^{7}$. Y ni que decir tiene que, ante dicha situación, la necesidad de coordinación entre los mecanismos existentes -tanto internos como internacionales- resulta imprescindible ${ }^{8}$. En este sentido se requiere, siguiendo el pensamiento del profesor PONS RAFOLS, un "planteamiento holístico y transversal para afrontar las cuestiones relacionadas con la salud global",

\footnotetext{
${ }^{6}$ Vid. S/RES/2243 (2015), de 14 de octubre de 2015, en la p. 3 de su parte preambular.

7 Vid. esta información en la Noticia del Servicio de Naciones Unidas titulada "Diferencias políticas, una docena de cascos azules y dos nuevos pozos en el norte de Haití", en http://www.un.org/spanish/News/story.asp?NewsID=35984\#.V 97KdSLRkg.
}

\footnotetext{
${ }^{8}$ Simplemente, valga como muestra un botón, como es la Resolución de la Asamblea General de Naciones Unidas de 18 de febrero de 2016, precedida de un buen número de resoluciones en sentido similar (A/RES/70/183), titulada "Salud mundial y política exterior: fortalecimiento de la gestión de las crisis sanitarias internacionales".

9 Vid. X. PONS RAFOLS, "International Law and Global Health: an Overview", 3 Paix et Sécurité Internationales (janvier-décembre 2015), pp. 33-60, en concreto p. 42.
} 
ámbito éste en el que el desarrollo, las catástrofes, las pandemias y la necesidad de control de las mismas conforman, nos atreveríamos a añadir, un círculo vicioso que cada vez ofrece una brecha más difícil de salvar si comparamos diversas regiones del planeta. Haití ofrece un ejemplo dramático en el que se han aunado todos esos elementos de manera fatídica.

Vayamos a los días previos a que el huracán Matthew arrasara el territorio haitiano. Sin ir más lejos, el 23 de septiembre de 2016, en su discurso ante la Asamblea General, el Presidente en funciones de Haití -J. Privertsubrayaba el sufrimiento de su pueblo por el azote de diversas epidemias, mencionando entre ellas el cólera, que se estaba recrudeciendo entonces. Asimismo, en su discurso realizó un llamamiento a la necesidad de lograr apoyo concreto y eficaz en materia de saneamiento para luchar contra dicha enfermedad, aludiendo al llamamiento que el propio Secretario General de Naciones Unidas había realizado ante el propio Consejo de Seguridad ${ }^{10}$.

¿Qué queremos señalar con ello? Simplemente un dato: parece que el cólera en Haití ha venido, junto con otro conjunto de enfermedades especialmente graves, lamentablemente, para quedarse. No son especulaciones: los fríos datos proporcionados por la Organización Mundial de la Salud hablan solos, si bien parece registrarse un importante descenso en el número de casos registrados de dicha enfermedad en los últimos años en el territorio haitiano ${ }^{11}: 179.379$ en 2010; un repunte muy significativo en 2011 con 340.311 casos, descendiendo significativamente desde entonces (112.076 en $2012,58.809$ en $2013,27.753$ en 2014), salvo en 2015, donde de nuevo la cifra ha crecido hasta 36.045 . Casi 9.000 casos más en 2015, conforme a los datos registrados respecto al año anterior hacen ver que la situación puede tener diversos calificativos, pero no el de éxito en lo que a la erradicación de la enfermedad respecta. Algo muy similar refleja el análisis del número de muertes registradas en Haití como consecuencia del cólera en los últimos años, que oscila entre los casi 4.000 fallecidos (3.990 en 2010), a los 296 en 2014, con un repunte en 2015 hasta alcanzar la cifra de 322 muertes a causa de esta enfermedad ${ }^{12}$.

\footnotetext{
${ }^{10}$ Véase a este respecto la p. 6 del Discurso pronunciado por el Presidente en funciones de Haití, en https://gadebate.un.org/sites/default/files/gastatements/71/71 HT fr.pdf. Algunos de sus párrafos más significativos serían los siguientes: "Durant les récentes années, mon peuple a énormement souffert d'une infestation galopante (...) par la malaria, le cholera, le zika, le chikungunya, la dengue et j'en passe (...) les déclarations de bonnes intentions, pour réconfortantes qu'elles soient, ne produisent aucun effet. Nous avons besoin d'un appui concret et efficace en matiére d'assainissement du milieu, en systéme d'eau potable, de traitement des ordures, etc... La recrudescence des cas de cholera ces derniers mois est l'un des défis qui posent aujourd'hui avec le plus d'acuité en Haïti. Elle illustre la détérioration sensible de la situation humanitaire, comme l'a souligné le Secrétaire général dans un récent rapport au Conseil de sécurité".

11 Vid. un registro de dicha información, por países, en http://apps.who.int/gho/data/node.main.175?lang=en.

12 Vid. a este respecto los datos suministrados por la OMS en http://apps.who.int/gho/data/node.main.176?lang=en.
} 
Resulta llamativo un hecho: si bien el cólera es una enfermedad que puede causar la muerte en cuestión de horas si no se trata -especialmente por deshidratación, a consecuencia de las diarreas agudas que puede llegar a producir- constituye una enfermedad cuyo tratamiento $-y$ en especial, la prevención- no debieran ser tan complejos, permitiendo así su erradicación ${ }^{13}$. Si no se trata, como pone de relieve la OMS, el riesgo de que la misma se expanda es muy elevado (de hecho, cada año se producen, según datos suministrados por la propia Organización, entre 1,4 y 4,3 millones de casos de cólera en el mundo y entre 28.000 y 142.000 defunciones). La existencia de una "Coalición Regional sobre Agua y Saneamiento para Eliminar la Transmisión del Cólera en La Española", pone de relieve la necesidad de cooperar para luchar contra su erradicación ${ }^{14}$.

Desde luego, una vista atrás pone de relieve un dramático dato: desde hace bastantes años, el cólera se ha implantado en Haití de manera estructural e incluso, lo que resulta aún más dramático, se observa un repunte de la enfermedad desde 2015, a juzgar por los datos que la OMS proporciona. Sin duda alguna, el cólera se ha instalado en el país, como el huracán Matthew pone de manifiesto, tema en el que nos detendremos en las líneas que siguen.

\section{El factor "coadyuvante": sobre el huracán Matthew y cuestiones aledañas}

La primera semana del mes de octubre de 2016 da comienzo con el paso de un devastador huracán -Matthew-, otro ejemplo más que permite acercarnos a una cruda realidad: la que se esconde tras un pernicioso triángulo compuesto por "pobreza extrema, desastres naturales y propagación de enfermedades que devienen endémicas y corren el riesgo de transformarse en verdaderas pandemias". Sin duda, éste puede ser el dibujo gráfico de la situación actual que vive Haití donde, tras el paso de Matthew se ha recrudecido aún más la propagación de esta enfermedad. Por supuesto, sin contar el número de fallecidos dejados a su paso -las cifras son dispares, entre más de 500 y casi novecientos dependiendo de las fuentes consultadas y los datos facilitados por las mismas- y la significativa cifra de afectados (que suman un par de millones) ${ }^{15}$.

\footnotetext{
${ }^{13}$ Como pone de manifiesto la OMS en la web dedicada a esta cuestión, hasta un $80 \%$ de los casos podrían tratarse con sales de rehidratación oral. Vid. una descripción de los principales rasgos de dicha enfermedad en http://www.who.int/mediacentre/factsheets/fs107/es/.

${ }^{14} \mathrm{~A}$ este respecto, véase la información y los esfuerzos que se intentan realizar en este sentido en http://www.paho.org/coleracoalicion/?lang=es.

${ }^{15}$ Vid. por ejemplo algunas de las referencias que facilitaban los medios de comunicación de esos

http://internacional.elpais.com/internacional/2016/10/06/actualidad/1475746470 475357.html; http://www.bbc.com/mundo/noticias-internacional-37591580; http://www.elmundo.es/internacional/2016/10/07/57f7718c46163f41718b463a.html.
} 
Dado lo caótico de la situación en estos momentos, a escasas semanas de haberse producido el paso del huracán por territorio haitiano, donde los cortes de carreteras y las situaciones de aislamiento que se viven en diversas zonas del país son considerables, nuestro objetivo en las líneas que siguen es enormemente modesto: analizar de manera inicial la actuación llevada a cabo por algunas de las Organizaciones Internacionales, agencias o fondos de Naciones Unidas, u otras relacionadas directamente con la región, con el objetivo de hacer frente a esta catástrofe y, de manera específica a la extensión del cólera. Como tuvimos ocasión de ver en el apartado anterior, la enfermedad, lejos de haberse erradicado antes de que este fenómeno tuviese lugar, había sufrido un incremento respecto de lo acaecido en los años inmediatamente anteriores.

A comienzos de esta década, en 2010, Ban Ki-moon declaraba "Haití no está solo", en referencia al terremoto que lo asoló entonces ${ }^{16}$. Seis años más tarde, la tragedia se ceba de nuevo con este país ${ }^{17}$. A mitad de octubre de este año 2016, el Secretario General de Naciones Unidas declaraba, en visita a Haití tras el paso del huracán: "La ONU está con ustedes"18. Sin duda alguna, buenos deseos, unidos al anuncio de creación de un Fondo Fiduciario para hacer frente a la catástrofe y al cólera de manera particular que tropiezan con una cruda realidad: la respuesta de la comunidad de donantes que, a fecha 18 de octubre solamente había contribuido con un $11 \%$ de los fondos que se preveían necesarios al llamamiento realizado desde la Organización ${ }^{19}$.

De manera más reciente -concretamente el 25 de octubre- el Centro de Noticias de Naciones Unidas informaba de algunas medidas que la Organización pretendía adoptar para afrontar la situación grave que vive Haití y, de manera particular, la epidemia de cólera ${ }^{20}$. "Forjar soluciones y programas de largo plazo" ${ }^{21}$, señala el vicesecretario de Naciones Unidas, dado que en este territorio, considerado como uno de los más pobres del planeta, la historia parece repetirse una y otra vez.

Una vez más, encontramos en este tema particular una palabra necesaria de manera general cuando nos enfrentamos a las catástrofes, a las pandemias resultado de las mismas y, en todo caso, a los dramas que se viven en el planeta del que el caso de Haití constituye un ejemplo paradigmático:

\footnotetext{
${ }^{16}$ Vid. http://www.un.org/es/sg/oped/2010/op-ed SG Haiti 19enero10.shtml, donde se recoge el artículo de opinión del Secretario General de la ONU traducido al español y publicado por el Washington Post el 19 de enero de 2010.

${ }^{17}$ Un dossier completo de las actuaciones de la ONU respecto de la situación de Haití puede leerse en http://www.un.org/spanish/News/focus.asp?focusID=17.

${ }^{18}$ Vid. http://www.un.org/spanish/News/story.asp?newsID=36065\#.WBsaMfrhCUk.

${ }^{19}$ Vid. http://www.un.org/spanish/News/story.asp?NewsID=36082\#.WBsj-PrhCUk.

${ }^{20}$ En palabras del vicesecretario general de la ONU, Jan Eliasson, eran necesarios 400 millones de dólares para hacer frente a la situación, tema que se encuentra sobre la mesa de la Asamblea General de Naciones Unidas en noviembre. Vid. http://www.un.org/spanish/News/story.asp?newsID=36134\#.WBskoPrhCUk.

${ }^{21}$ Vid. http://www.un.org/spanish/News/story.asp?NewsID=36062\#.WBsI7 rhCUk.
} 
resulta imprescindible una coordinación de esfuerzos de la comunidad internacional ${ }^{22}$ en su conjunto para hacer frente a estas dramáticas situaciones. Esa idea de comunidad, en palabras de GARCíA SAN JOSÉ ${ }^{23}$, basada en la soberanía responsable y en el principio de rendición de cuentas habría de ser un paradigma a seguir, que permitiría asumir una mayor responsabilidad respecto a los retos que este siglo XXI muestra. Hacer frente a situaciones como la haitiana constituye un buen ejemplo de ello.

Varios son los retos que han de afrontarse desde el contexto universal y onusiano en particular- frente a la "crisis" que ya parece estructural en Haití. Como en toda situación de esta índole, al menos tres son las fases a tener en cuenta con el objetivo de hacer frente a dicha situación: prevención, preparación y respuesta, junto con coordinación y coherencia ${ }^{24}$. A este respecto, son varios los organismos internacionales implicados en la lucha contra el cólera en las últimas semanas, como continuación de una labor emprendida ya hace años. No en vano, desde hace ya mucho tiempo, en Naciones Unidas existe un Coordinador Principal de la Respuesta al Cólera en Haití, aspecto que implica hacer de la necesidad, virtud, sin duda alguna. Cabe citar, por ejemplo, la Resolución del Consejo de Seguridad de 13 de octubre de $2016^{25}$, si bien centrada en el futuro de la MINUSTAH y en la puesta en práctica de medidas que mejoren la estabilidad en Haití, que contiene referencias en su parte preambular a la expansión del cólera que sufre este país. Sin ninguna duda destaca además esa visión holística de la seguridad

${ }^{22}$ Cuando nos referimos a la comunidad internacional lo hacemos de manera general respecto a la necesidad de colaboración de todos los sujetos implicados (sean estos Estados, Organizaciones Internacionales, ONG's u otros actores que pudiesen prestar su asistencia ante situaciones como la descrita, ocasionada por el paso del huracán). En este sentido resultan curiosas las actuaciones que, por ejemplo, la Unión Europea ha desempeñado en este terreno, y las que quedan por venir; sobre este particular vid. M.I. TORRES CAZORLA, "¿Lecciones aprendidas? El papel de la Unión Europea en materia de prevención y coordinación en caso de catástrofe", en J.M. SÁNCHEZ PATRONN, M.I. TORRES CAZORLA y D. GARCÍA SAN JOSÉ, Bioderecho, Seguridad y Medioambiente. Biolaw, Security and Environment, Tirant lo Blanch, Valencia, 2015, pp. 151-171.

${ }^{23}$ Vid. D.I. GARCIA SAN JOSÉ, "Seguridad medioambiental y principio de necesidad en Bioderecho Internacional", en Bioderecho, Seguridad y Medioambiente, op. cit., pp. 131-150, en particular pp. 134-137.

${ }^{24}$ De algún modo asumimos las ideas expresadas por A. BAUTISTA HERNÁEZ, en "Recientes avances en la regulación de la Unión Europea en materia de catástrofes: el Mecanismo de Protección Civil de la Unión", Bioderecho, Seguridad y Medioambiente, op. cit., pp. 173-194, en particular pp. 185-191.

${ }^{25}$ S/RES/2313 (2016). En la misma se reconoce que "los desafíos que enfrenta Haití están interconectados", y se observa "un aumento en los presuntos casos de cólera y las muertes relacionadas" con dicha enfermedad. Se hace eco de los esfuerzos que tanto el Gobierno haitiano como la MINUSTAH y los organismos de Naciones Unidas que apoyan el Plan Nacional para la Eliminación del Cólera están realizando; del mismo modo, recalca "la importancia de fortalecer las instituciones del sistema sanitario nacional de Haití" y reconoce "los esfuerzos de las Naciones Unidas para combatir el cólera, concretamente mediante la iniciativa del Secretario General de apoyo al Plan Nacional para la Eliminación del Cólera". Igualmente, toma nota "de la intención del Secretario General de preparar un paquete de medidas para ofrecer apoyo y asistencia material a los haitianos directamente afectados por el cólera". 
que, de manera específica se pone de relieve en Haití, en los siguientes términos:

“...reiterando la necesidad de que la seguridad vaya acompañada del desarrollo económico y social, incluidas actividades de reducción de riesgos y preparación que tengan en cuenta la extrema vulnerabilidad del país a los desastres naturales, actividades en las que el Gobierno de Haití desempeña un papel rector"26.

Diferenciaremos seguidamente la labor emprendida por algunos de los principales organismos respecto al último rebrote del cólera en Haití, teniendo presente que estos datos son muy recientes y que los mismos pueden sufrir cambios de manera constante en cualquier momento:

\section{a) El papel de las Organizaciones Internacionales de la "familia onusiana": el caso específico de la Organización Mundial de la Salud (OMS)}

Una de las Organizaciones Internacionales más implicadas en esta situación -como no podría ser de otra forma- es la Organización Mundial de la Salud, organismo especializado de Naciones Unidas creado en 1946, una de cuyas principales funciones consiste precisamente en la lucha contra la propagación de enfermedades infecciosas ${ }^{27}$. A ella nos hemos referido ya con anterioridad aludiendo a sus estudios en relación con el tema que nos ocupa.

Como medida operacional, la OMS, en contacto directo con las autoridades locales -concretamente del Ministerio de Salud de Haití- y con el patrocinio de la Organización Panamericana de la Salud (brazo de la OMS en la región) va a poner en marcha a comienzos de este mes de noviembre una campaña de vacunación con el objetivo de controlar la enfermedad. Se trata de llevar un millón de dosis de vacuna oral a las zonas más afectadas del país por el paso del huracán (especialmente en los departamentos del sur de Grand 'Anse y Sud ${ }^{28}$. Asimismo, dicha labor cuenta con la implicación de otros entes internacionales que desempeñan tareas en Haití como UNICEF, GHESKIO, Partners in Health, los Centros para el Control y la Prevención de Enfermedades (CDC), o International Medical Corps, entre otros. Destaca además toda la labor divulgativa y de información que la OMS lleva a cabo con

\footnotetext{
${ }^{26}$ Vid. S/RES/2313 (2016), en su parte preambular, pp. 3-4.

${ }^{27}$ Sobre el particular, vid. X. PONS RAFOLS, "International Law and Global Health...", loc. cit., pp. 45-47, así como, del mismo autor, "La Organización Mundial de la Salud y la cooperación internacional frente a las grandes pandemias: el nuevo Reglamento Sanitario Internacional", en X. PONS RAFOLS (ed.), Salud pública mundial y derecho internacional, Marcial Pons, Madrid, 2010, pp. 165-192.
} 
DOI: http://dx.doi.org/10.12795/IETSCIENTIA.2016.i02.10

el objetivo de informar, prevenir, luchar contra su expansión y tratar de erradicar las enfermedades y, de manera especial, el cólera entre ellas ${ }^{29}$.

\section{b) Otros entes vinculados a la Organización de Naciones Unidas que desempeñan un papel relevante en la lucha contra el cólera en Haití}

En este apartado señalaremos algunas de las actividades desempeñadas por programas y agencias vinculadas a la ONU que concentran esfuerzos en luchar contra el cólera tras el paso del huracán. Por ejemplo, el Programa Mundial de Alimentos (PMA o WFP, en sus siglas en inglés) centra sus esfuerzos en la seguridad alimentaria en la zona ${ }^{30}$. El Fondo de Naciones Unidas para la Infancia (UNICEF) ha puesto en práctica diversas actuaciones, entre las que destacan estaciones potabilizadoras, participación en la campaña de vacunación contra el cólera, así como diversos kits de prevención contra la enfermedad, poniendo de relieve la existencia de más de 600.000 niños en situación de vulnerabilidad tras el paso del huracán ${ }^{31}$. En consonancia con ello, el Fondo Central para la Acción en Casos de Emergencia (CERF) ha destinado 9 millones de dólares a dicha tarea ${ }^{32}$. Asimismo, la OCHA (Oficina de la ONU para la Coordinación de Asuntos Humanitarios) ha venido alertando desde tiempo atrás del repunte en los casos de la enfermedad registrados en Haití (prácticamente desde junio de 2015) ${ }^{33}$. En la actualidad se han llevado a cabo numerosas actuaciones, en particular en una de las regiones más afectadas por el huracán Matthew: la zona de Les Cayes ${ }^{34}$. Como medida operacional específica, la colaboración del personal de la Cruz Roja sobre el terreno implicado en la campaña de vacunación contra el cólera debe destacarse ${ }^{35}$.

29 A este respecto, vid. http://www.who.int/features/factfiles/chttps://www.humanitarianresponse.info/operations/haitihol era/es/, donde se contiene diez datos fundamentales sobre el cólera a tener muy en cuenta.

30 Un mapa ejemplificativo de la labor de asistencia alimentaria en el país véase en https://www.humanitarianresponse.info/en/system/files/documents/files/hti wfpo fooddistributio n a3l 20161101.pdf; igualmente, de manera complementaria en https:/www.humanitarianresponse.info/en/system/files/documents/files/securite alimentaire 3w op $31102016 \mathrm{fr}$ 0.pdf.

${ }^{31} \mathrm{Al}$ respecto, véase http://reliefweb.int/report/haiti/haiti-un-mois-apr-s-le-passage-de-matthew600000-enfants-ont-toujours-besoin-daide.

32 Vid. http://www.unocha.org/cerf/resources/top-stories/cerf-allocates-funds-kick-startassistance-following-hurricane-matthew-and.

Vid.

información

en

http://www.un.org/spanish/News/story.asp?NewsID=34232\#.WBySvPrhCUk.

34 Vid. sobre ello la profusa información que se suministra en https://www.humanitarianresponse.info/operations/haiti. Los efectos del huracán y sus consecuencias en lo relativo a la expansión de la enfermedad resultan visibles en el siguiente esquema explicativo https://www.humanitarianresponse.info/en/system/files/documents/files/haiti snapshot 21 oct 16.pdf.

35 Vid. http://reliefweb.int/report/haiti/haiti-red-cross-volunteers-prepare-cholera-vaccinationcampaign. 
Finalizamos con una breve reflexión, para la que no dudamos en reproducir las palabras del profesor RODRíGUEZ CARRIÓN que hace casi dos décadas ya nos alertaba de una situación que resulta plenamente aplicable a nuestros días y de modo particular a una realidad tan dramática como la que vive Haití. Nuestro maestro denunciaba entonces que "quizás estemos viviendo un derecho internacional que posiblemente esté perdiendo el reto de la solidaridad para acentuar desigualdades inaceptables, desigualdades cuya misma existencia es negada o estimada como solucionable con la adopción de recetas que en el primer mundo dieron obvio y óptimo resultado",36. Esta visión crítica la manifestaban en sentido similar GARCÍA MATAMOROS y ABELLÓ GALVIS al plantear que "el derecho internacional no puede permanecer inmóvil $y$ en ese sentido resulta necesario volver a pensar en el derecho al desarrollo como una propuesta integradora, internacional y humana (...) vale la pena la utopía y no perder la fe en la potencialidad del derecho internacional”37. Abramos un ventanuco a la esperanza, a pesar de que los indicios no dan muestras loables de que la situación que vive Haití pueda resolverse de modo sencillo en pocos años, siendo realistas.

\section{BIBLIOGRAFÍA CITADA}

BAUTISTA HERNÁEZ, A., "Recientes avances en la regulación de la Unión Europea en materia de catástrofes: el Mecanismo de Protección Civil de la Unión”, en J.M. SÁNCHEZ PATRÓN, M.I. TORRES CAZORLA y D. GARCÍA SAN JOSÉ, Bioderecho, Seguridad y Medioambiente. Biolaw, Security and Environment, Tirant lo Blanch, Valencia, 2015, pp. 173194.

BODE, T.G., "Cholera in Haiti: United Nations Immunity and Accountability", 47 Georgetown Journal of International Law (2016-2), pp. 759-791

GARCÍA MATAMOROS, L.V., y ABELLO GALVIS, R., "Reflexiones sobre el papel del derecho al desarrollo en las relaciones entre paz, seguridad y desarrollo", en La seguridad internacional en el siglo XXI, op. cit., pp. 167-188.

GARCIA SAN JOSÉ, D.I., "Seguridad medioambiental y principio de necesidad en Bioderecho Internacional”, en Bioderecho, Seguridad y Medioambiente, op. cit., pp. 131-150.

HOLLENBERG, S., "Immunity of the UN in the case of Haitian Cholera Victims", 19 Journal of International Peacekeeping (2015, 1 y 2), pp. 118-141.

MÉGRET, F., "La responsabilité des Nations Unies aux temps du chólera”, 47 Revue Belge de Droit International (2013-1), pp. 161-189.

PAVONI, R., "Choleric notes on the Haiti Cholera Case", Questions of International Law (27 July 2015), pp. 11-37, accesible en http://www.qil-qdi.org/choleric-notes-on-the-haiti-choleracase/.

\footnotetext{
${ }^{36}$ Podría parecer pesimista dicha visión que, sin embargo, ofrecía un rayo de luz al señalar que "no hay nada escrito, salvo la página que cada día escribimos"; vid. A.J. RODRíGUEZ CARRIÓN, El Derecho Internacional en el Umbral del siglo XXI, Universidad de Málaga, SPICUM, Málaga, 1999, p. 74.

${ }^{37}$ Vid. L.V. GARCíA MATAMOROS y R. ABELLO GALVIS, "Reflexiones sobre el papel del derecho al desarrollo en las relaciones entre paz, seguridad y desarrollo", en La seguridad internacional en el siglo XXI, op. cit., pp. 167-188, en particular p. 188.
} 
PONS RAFOLS, X., "International Law and Global Health: an Overview", 3 Paix et Sécurité Internationales (janvier-décembre 2015), pp. 33-60; "La Organización Mundial de la Salud y la cooperación internacional frente a las grandes pandemias: el nuevo Reglamento Sanitario Internacional", en X. PONS RAFOLS (ed.), Salud pública mundial y derecho internacional, Marcial Pons, Madrid, 2010, pp. 165-192.

RODRÍGUEZ CARRIÓN, A.J., El Derecho Internacional en el Umbral del siglo XXI, Universidad de Málaga, SPICUM, Málaga, 1999.

TAYLOR, K.N., "Shifting Demands in International Institutional Law: Securing the United Nations' Accountability for the Haitian Cholera Outbreak", 45 Netherlands Yearbook of International Law (2014), pp. 157-195.

TORRES CAZORLA, M.I., "¿Lecciones aprendidas? El papel de la Unión Europea en materia de prevención y coordinación en caso de catástrofe", en Bioderecho, Seguridad y Medioambiente, op. cit., pp. 151-171; "Environmental Security: an Initial View from the Perspective of International Law", en La seguridad internacional en el Siglo XXI. Nuevas Perspectivas, E.M GARCÍA RICO y M.I. TORRES CAZORLA (coords.), Plaza y Valdés, Madrid, 2011, pp. 145-166.

VON EINSIEDEL, S., y MALONE, D.M., "Haiti", en The UN Security Council. From the Cold War to the 21st Century, D.M. MALONE (ed.), Lynne Rienner Publishers, Boulder, London, 2004, pp. 467-482. 